\title{
REPORTE DE CASO: TRASPLANTE RENAL EN UN PACIENTE CON EPIDERMOLISIS BULLOSA.
}

\section{RENAL TRANSPLANTATION IN A PATIENT WITH RECESSIVE DYSTROPHIC EPIDERMOLYSIS BULLOSA: A CASE REPORT}

\author{
Emanuel José Saad ${ }^{1,2,3}$, Ricardo Arturo Albertini ${ }^{1,2}$, Carlos Chiurchiu ${ }^{4}$, Pablo Ulises Massarit, Jorge De La \\ Fuente ${ }^{2,4}$.
}

\section{Resumen:}

La epidermolisis bullosa distrófica recesiva (EBDR) es una genodermatosis extremadamente infrecuente, caracterizada por la existencia de alteraciones a nivel de las fibras de anclaje que unen la membrana basal de la epidermis a las estructuras subyacentes. Un elemento característico de esta entidad es la formación recurrente de ampollas en piel y mucosas ante traumatismos mínimos, con posterior cicatrización. Los pacientes con EBRD frecuentemente desarrollan enfermedad renal crónica y requieren de terapia de reemplazo renal, constituyendo una importante causa de morbilidad y mortalidad en estos pacientes. El rol del trasplante renal es poco conocido en este tipo de pacientes.

Se presenta el caso de un paciente con enfermedad renal terminal y EBDR que es tratado con trasplante renal y su seguimiento a lo largo de un período de 83 meses luego del trasplante. Durante dicho período se observó una baja frecuencia de intercurrencias infecciosas, así como la ausencia de desarrollo de neoplasias cutáneas. El trasplante renal podría ser una alternativa a la terapia de reemplazo dialítica en los pacientes con epidermolisis bullosa asociada a enfermedad renal terminal, reduciendo las comorbilidades asociadas a las terapias dialíticas.

Palabras clave: epidermólisis ampollosa; epidermólisis ampollosa distrófica; trasplante de riñón.

\begin{abstract}
:
Recessive dystrophic epidermolysis bullosa (RDEB) is a rare genodermatosis characterized by abnormalities in the anchoring fibrils which attach the basal cell layer of the epidermis to the underlying structures. A characteristic feature of this disorder is the presence of recurrent blistering or erosions, the result of even minor traction to these tissues. Patients with RDEB frequently develop chronic renal failure, and require renal replacement therapy being a major cause of morbidity and mortality. The role of renal transplantation in these patients is scarcely known.

We present the case of an end-stage renal disease patient with RDEB treated by renal transplantation and his follow-up during a period of 83 months after the transplant. In this period, there were very low frequency of serious infections as well as the absence of skin tumors.

Renal transplantation could be an alternative to renal replacement therapy in epidermolysis bullosa patients with end-stage renal disease, reducing the comorbidities associated with this treatment.
\end{abstract}

Keywords: epidermolysis bullosa; epidermolysis bullosa dystrophica; kidney transplantation.

\footnotetext{
1 Departamento de Medicina Interna, Hospital Privado Universitario de Córdoba,

2 Instituto Universitario de Ciencias Biomédicas de Córdoba

3 Email de contacto: emanuelsaad@hotmail.com

4 Departamento de Nefrología, Hospital Privado Universitario de Córdoba, Argentina
} 


\section{Introducción}

La epidermólisis bullosa (EB) hereditaria es una genodermatosis extremadamente infrecuente, caracterizada por la existencia de alteraciones a nivel de la unión dermo-epidérmica que favorecen la formación de ampollas en piel y mucosas ante traumatismos mínimos ${ }^{[1]}$. Según el nivel donde se produce dicha alteración, es posible distinguir 4 tipos principales de EB: EB Simple (capa basal de la epidermis), EB de la unión (membrana basal), EB distrófica (fibras de anclaje que unen la membrana basal a la dermis) y el recientemente añadido síndrome de Kindler (alteraciones en varios niveles de modo simultáneo) ${ }^{[2]}$. La epidermolisis bullosa distrófica (EBD) puede ser autosómica dominante o recesiva ${ }^{[2]}$, caracterizándose sus formas generalizadas por la presencia de lesiones de piel en sitios expuestos a traumatismos repetitivos y lesiones a nivel de mucosas. La afectación de las mucosas del tracto digestivo puede conducir a un síndrome de malabsorción y consiguiente estado de desnutrición calórico-proteico. Así mismo, estos pacientes presentan una mayor predisposición al desarrollo de carcinoma escamoso invasivo que la población en general, teniendo un comportamiento más agresivo ${ }^{[3]}$. El desarrollo de enfermedad renal crónica (ERC) es muy frecuente en este grupo de pacientes, siendo generalmente secundario a hidronefrosis, glomerulonefritis post-estreptocócica, enfermedad mesangial por $\lg \mathrm{A}$ o amiloidosis ${ }^{[4]}$. Por dicho motivo, muchos de ellos requieren inicio de terapia de reemplazo renal (TRR), a través de hemodiálisis o diálisis peritoneal. Sin embargo, el rol del trasplante renal es escasamente conocido en los pacientes con EBD ${ }^{[5]}$.

Presentamos el caso de un paciente con EB distrófica recesiva (EBDR) con ERC terminal, que fue tratado con trasplante renal y su seguimiento posterior al trasplante. Se ha obtenido el consentimiento informado del paciente.

\section{Exposición de caso clínico}

Paciente de sexo masculino, con diagnóstico al momento de su nacimiento de EBDR. A lo largo de su vida presentó múltiples lesiones ampollares en piel y mucosas que curaron con cicatrices deformantes. Desarrolló estenosis esofágica, asociado a un estado de desnutrición calórico-proteica crónico. A los 9 años de edad se diagnosticó ERC de etiología indeterminada, con necesidad de inicio de TRR a los 16 años de edad. Inicialmente realizó diálisis peritoneal durante 3 años, seguido de hemodiálisis durante 3 meses. Durante este período el paciente presentó recurrentes episodios de infecciones urinarias y peritonitis bacterianas asociadas a la diálisis peritoneal. A los 19 años de edad, el equipo médico tratante realizó una valoración interdisciplinaria en conjunto con el paciente y su familia y decidieron realizar un procedimiento de trasplante renal, que se desarrolló en junio del año 2008. Se seleccionó un donante vivo relacionado, con un match de HLA A/B/DR de 2/1/1 respectivamente y cross match del receptor del $5 \%$. El procedimiento quirúrgico se desarrolló sin complicaciones, presentando buena evolución en postoperatorio inmediato con función espontánea de injerto, sin requerimiento de TRR. El esquema inmunosupresor inicial prescripto incluyó azatioprina, ciclosporina y esteroides, recibiendo como inducción gammaglobulina humana. Como profilaxis infecciosa, se utilizó trimetoprima-sulfametoxazol durante el primer año y aciclovir en forma crónica.

El paciente evolucionó favorablemente en los meses posteriores al trasplante (valor de creatinina a los 3 meses post-trasplante de $0,85 \mathrm{mg} / \mathrm{dL}$ y realación proteinuria: creatininuria de 0,74$)$. No hubo cambios significativos respecto a la formación y evolución de las lesiones ampollares respecto al período pretrasplante. A los 28 meses post trasplante, el paciente presentó un deterioro progresivo de la función renal e incremento de proteinuria (creatinina de $1,7 \mathrm{mg} / \mathrm{dL}$ y realación proteinuria: creatininuria de 1,90 ). Se realizó una biopsia renal, cuya anatomía patológica informó nefropatía crónica del injerto grado II de Banff, C4d negativo. Continuó con función renal estable, sin requerimiento de terapia de reemplazo renal dialítico.

El primer evento de infeccioso significativo fue un cuadro de celulitis leve sin síntomas sistémicos, a los 40 meses del trasplante. Consecuentemente, se cambió el esquema inmunosupresor reemplazando azatioprina por micofenolato sódico, continuando con ciclosporina y corticosteroides. Entre otros episodios significativos se destacaron un episodio de meningitis aséptica (51 meses post-trasplante), una neumonía adquirida en la comunidad y un episodio de infección cutánea localizada (60 meses post trasplante). Se realizó el hallazgo incidental de un aneurisma sacular en sifón carotídeo asintomático no complicado, en contexto de una urgencia hipertensiva (62 meses post trasplante). Requirió de varios procedimientos de dilataciones esofágicas, consecuencia de su enfermedad de base.

A los 75 meses post-trasplante presentó un cuadro de neumonía grave asociado a cuidados de la salud, con requerimiento de internación en unidad de cuidados intensivos y tratamiento con antibióticos de amplio espectro y anfotericina B por sospecha de infección micótica. En este contexto presentó un importante deterioro de la función renal, requiriendo TRR con hemodiálisis de modo definitivo. El paciente evolucionó de modo favorable con el tratamiento antimicrobiano instaurado. Luego de dicho 
episodio, el paciente no desarrolló nuevas complicaciones o intercurrencias infecciosas hasta el mes 83 de post trasplante. Durante todo el período de seguimiento el paciente fue controlado frecuentemente por el servicio de dermatología y nefrología de nuestro hospital. Así mimo, durante dicho período no se detectaron indicios de lesiones sugestivas de malignidad.

\section{Discusión}

El presente caso representó un gran desafío para disminuir la morbimortalidad de la ECR con TRR en un paciente con EBDR. El trasplante renal constituye una estrategia conocida para ello, pero la utilización de los inmunosupresores se puede asociar a múltiples eventos adversos de índole infecciosa y neoplásica. Los pacientes con EB poseen un estado basal de inmunosupresión multifactorial, debido a la existencia de alteraciones de la barrera cutáneo-mucosa y la disminución de las poblaciones de linfocitos T helper CD4 y NK, secundario al estado de desnutrición calórico-proteica ${ }^{[6]}$. Además, cerca del $50-80 \%$ de los pacientes con EBDR, desarrollan a lo largo de su vida carcinoma cutáneo de células escamosas (especialmente luego de la segunda década de vida) ${ }^{[3]}$.

El desarrollo de ERC es una de las complicaciones más frecuentes de los pacientes con EB, siendo responsable del $12 \%$ de las muertes de los mismos ${ }^{[7]}$. Los pacientes con EBDR y ERC terminal generalmente son tratados con hemodiálisis o diálisis peritoneal como TRR, siendo poco conocido el rol del trasplante renal en los mismos ${ }^{[5]}$. El trasplante renal, es la mejor opción para los pacientes con ERC terminal en general, sin embargo, la utilización prolongada de drogas inmunosupresoras podría estar asociada a un incremento de infecciones oportunistas y neoplasias malignas de piel y mucosas. Se ha observado según diferentes reportes que cerca del 60-90\% de los trasplantados renales, presentan algún tipo de lesiones cutáneas asociadas al tratamiento inmunosupresor ${ }^{[8]}$. Las lesiones más frecuentemente descriptas son de causa infecciosa (principalmente en los primeros 60 meses post-trasplante), seguidas de las reacciones adversas de los fármacos inmunosupresores y por ultimo lesiones malignas y premalignas (generalmente luego de los 60 meses post-trasplante). Las infecciones verrucosas virales y dermatofitosis son las causas más frecuentes de infecciones cutáneas en este grupo de pacientes, mientras que las bacterianas presentan baja prevalencia ${ }^{[9]}$. Se ha descripto que la utilización de azatioprina en este contexto, produciría una reducción de células de Langerhans en la epidermis, lo cual podría explicar la mayor susceptibilidad a infecciones fúngicas ${ }^{[10]}$.

Varios estudios han reportado que la utilización de terapia inmunosupresora en pacientes trasplantados renales incrementa la incidencia de neoplasias, principalmente de piel. Wimmer et al, reportaron en un estudio realizado en Alemania una incidencia acumulativa de cáncer de piel no melanocítico en trasplantados renales cercana al $20.5 \%{ }^{[11]}$, mientras Kasiske et al, en Estados Unidos de América han documentado una incidencia acumulativa de $7 \%$ luego de 3 años de inmunosupresión ${ }^{[12]}$. Respecto a estudios latinoamericanos, Dufrechou et al, han reportado en Uruguay que el $60 \%$ de los pacientes trasplantados renales presentaron al menos 1 manifestación cutánea, de las cuales el 14.4\% eran lesiones malignas y premalignas ${ }^{[9]}$. Dentro de los inmunosupresores, se ha observado que azatioprina se encuentra asociado a la aparición de carcinomas escamosos múltiples, probablemente relacionado al efecto inmunosupresor y la foto-activación de su núcleo aromático con luz ultravioleta ${ }^{[13]}$.

Existe poca evidencia sobre la utilización de terapia inmunosupresora en pacientes con epidermólisis bullosa. Se ha reportado un solo caso de trasplante renal en pacientes con EB, donde se describe la evolución favorable durante los primeros 5 meses post trasplante, de un paciente de origen chino de 33 años con EB juntional No-Herlitz ${ }^{[5]}$. Otros tipos de trasplantes reportados en estos pacientes son el trasplante alogénico de piel con fines reconstructivos ${ }^{[14]}$, y trasplante alogénico de médula ósea con el objetivo terapéutico de logara la producción de Colágeno VII en la lámina densa, para mejorar la evolución de la enfermedad ${ }^{[15]}$.

\section{Conclusión}

La importancia del presente caso radica en la posibilidad de describir la evolución prologada de un paciente con EBDR que ha recibido un trasplante renal. Durante un seguimiento de 83 meses posttrasplante, se observó una baja frecuencia de intercurrencias infecciosas (las cuales fueron leves, excepto un solo cuadro de neumonía grave), así como la ausencia de desarrollo de neoplasias cutáneas. Sin embargo, a los 75 meses post trasplante, el paciente debe ingresar nuevamente a TRR por deterioro de la función del implante renal. Este caso podría alentar a futuras investigaciones para considerar al trasplante renal como una alternativa para disminuir las comorbilidades asociadas a la terapia dialítica en pacientes con EB y ERC terminal. 


\section{Bibliografía}

1. LeBleu VS, Macdonald B, Kalluri R. Structure and function of basement membranes. Exp Biol Med (Maywood). 2007;232(9):1121-29. [PubMed]

2. Fine JD, Eady RA, Bauer EA, Bauer JW, Bruckner-Tuderman L, et al. The classification of inherited epidermolysis bullosa (EB): Report of the Third International Consensus Meeting on Diagnosis and Classification of EB. J Am Acad Dermatol. 2008;58(6):931-50. [PubMed]

3. Fine JD, Johnson LB, Weiner M, Li KP, Suchindran C. Epidermolysis bullosa and the risk of life-threatening cancers: The National EB Registry experience, 1986-2006. J Am Acad Dermatol. 2009;60(2):203-11. [PubMed]

4. Fine JD, Johnson LB, Weiner M, Stein A, Cash S, et al. Inherited epidermolysis bullosa and the risk of death from renal disease: experience of the National Epidermolysis Bullosa Registry. Am J Kidney Dis. 2004;44(4):651-60. [PubMed]

5. Ungureanu S, Adni T, Brown T, Inston N, Heagerty A. Successful renal transplant in a patient with non-Herlitz junctional epidermolysis bullosa. Clin Exp Dermatol. 2014;39(3):330-2. [PubMed]

6. Marinkovich MP. Inherited Epidermolysis Bullosa. In: Fitzpatrick's Dermatology in General Medicine. 8th ed. United State of America: Mc Graw Hill; 2012. P. 649-65.

7. Boeira VL, Souza ES, Rocha Bde O, Oliveira PD, Oliveira Mde F, et al. Inherited epidermolysis bullosa: clinical and therapeutic aspects. An Bras Dermatol. 2013;88(2):185-98. [PubMed]

8. Castello M, Gregorini M, Rampino T, Bosio F, Bedino G, et al. A retrospective analysis of dermatological lesions in kidney transplant patients. Indian J Med Res. 2013;137(6):1188-92. [PubMed]

9. Zamanian A, Mahjub H, Mehralian A. Skin diseases in kidney transplant recipients. Urol J. 2006;3(4):230-3. [PubMed]

10. Bergfelt L, Larko O, Blohmé I. Skin disease in immunosuppressed patients in relation to epidermal Langerhans' cells. Acta Derm Venereol. 1993;73(5):330-4. [PubMed]

11. Wimmer CD, Rentsch M, Crispin A, Illner WD, Arbogast $H$, et al. The janus face of immunosuppression - de novo malignancy after renal transplantation: the experience of the Transplantation Center Munich. Kidney Int. 2007;71(12):1271-8. [PubMed]

12. Kasiske BL, Snyder JJ, Gilbertson DT, Wang C. Cancer after kidney transplantation in the United States. Am J Transplant. 2004;4(6):905-13. [PubMed]

13. Ulrich C, Arnold R, Frei U, Hetzer R, Neuhaus $P$, et al. Skin changes following organ transplantation: an interdisciplinary challenge. Dtsch Arztebl Int. 2014;111(11):188-94. [PubMed]

14. Buonocore SD, Ariyan S. Cadaveric allograft for wound closure after resection of squamous cell carcinoma in patients with recessive dystrophic epidermolysis bullosa: a report of 32 resections and repairs in 2 patients. Ann Plast Surg. 2009;63(3):297-9. [PubMed]

15. Wagner JE, Ishida-Yamamoto A, McGrath JA, Hordinsky M, Keene DR, et al. Bone marrow transplantation for recessive dystrophic epidermolysis bullosa. N Engl J Med. 2010;363(7):629-39. [PubMed] 